REVIEW

\title{
Aneuploidy and malignancy: an unsolved equation
}

\section{P Dey}

J Clin Pathol 2004;57:1245-1249. doi: 10.1136/icp.2004.018952

Aneuploidy is frequently noted in malignant tumours. There is much controversy about its cause and effect in relation to malignant tumours. Failure of the spindle checkpoint caused by mutation of the responsible genes may be one of the important factors for the development of aneuploidy. Telomere dysfunction may also be a possible source of failure of cytokinesis resulting in aneuploidy. Evidence such as tumour specific aneuploidy, presence of aneuploidy in various preneoplastic conditions, increased frequency of genetic instability in aneuploid cell lines compared with diploid cells, and mutation of mitotic checkpoint genes suggests that aneuploidy possibly plays an active role in carcinogenesis. In this brief review, the various aspects of aneuploidy with special emphasis on its mechanism of development and impact on progression of cancer are discussed.
Correspondence to: Dr P Dey, Pathology Department, Kuwait Cancer Control Centre, PO Box 42262, 70653 Shuwaikh, Kuwait; deypranab@hotmail.com

Accepted for publication 11 June 2004
$\mathrm{T}$ he loss or gain of one or more chromosomes is known as aneuploidy. Normal human somatic cells have 23 pairs of chromosomes. Human cells with a chromosome number different from 46 or with an abnormal complement of chromosomes that add up to 46 are aneuploid. This imbalance of chromosome number may be in germ cells or somatic cells. Down's syndrome, in which there is trisomy $2 \mathrm{l}$, is an example of germ cell aneuploidy. The Philadelphia chromosome in chronic granulocytic leukaemia was studied in detail with the help of traditional metaphase cytogenetics. ${ }^{1}$ The Philadelphia chromosome is defined as translocation of chromosome 9 and $22,{ }^{2}$ and this structural chromosomal change provided new insight into the pathogenesis of leukaemia. However simple structural changes without numerical chromosomal aberration should not be considered as aneuploidy. Aneuploidy has frequently been noted in many solid tumours, and occasionally a few uterine carcinoma and prostatic carcinomas have been reported to be diploid (table 1). ${ }^{3-14}$ Despite the presence of a high frequency of aneuploidy in cancer, we still do not know the exact role of aneuploidy in carcinogenesis. Is it simply a nonspecific change during tumorigenesis or is it actively involved in the development and progression of tumours? What causes normal cells to become aneuploid? We still do not know the exact answers to these questions and there is much controversy about the cause and effect of aneuploidy related to carcinogenesis. ${ }^{15-17}$ In this brief review, the possible mechanisms responsible for aneuploidy and the role of aneuploidy in malignancy are discussed.

"Despite the presence of a high frequency of
aneuploidy in cancer, we still do not know the
exact role of aneuploidy in carcinogenesis"

\section{TYPES OF ANEUPLOIDY}

In the case of aneuploidy, the cell may gain or lose one or more chromosomes. Cells with less than a diploid chromosome content are referred to as having hypodiploid aneuploidy. This is an uncommon finding in malignancy because hypodiploid cells rarely survive. Cells with more than a diploid but less than a tetraploid complement of chromosomes are referred to as having hyperdiploid aneuploidy.

The DNA index of a tumour indicates the degree of aneuploidy and it is calculated as modal number of chromosomes of the tumour population divided by the reference number of chromosomes of the normal diploid cells. Thus, the DNA index of a diploid tumour is 1 , a hypodiploid tumour is less than $\mathrm{l}$, and a hyperdiploid tumour is 1 to 2 .

\section{DETECTION OF ANEUPLOIDY}

Aneuploidy can be detected with the help of traditional metaphase cytogenetics, interphase cytogenetics (fluorescent in situ hybridisation (FISH), multicolour FISH, spectral karyotyping, and comparative genomic hybridisation techniques (CGH)), flow cytometry (FCM), and image cytometry (ICM). FCM and ICM can measure the relative DNA content of the cell with respect to reference diploid cells.

\section{TRADITIONAL METAPHASE CYTOGENETICS}

Chromosomes are traditionally analysed by the chromosomal banding technique, which studies the chromosomes in a metaphase spread of a dividing cell population. The cell population is collected from the target tissue such as blood or bone marrow. Cell division is stimulated artificially by phytohaemagglutinin and the cell suspension is grown in a culture medium. To arrest as many cells as possible in metaphase, the formation of the mitotic spindle is prevented by a drug with a cholchicine-like effect (a spindle inhibitor). The cells are treated with hypotonic solution and a chromosome preparation is made.

Abbreviations: APC, anaphase promoting complex; $\mathrm{CGH}$, comparative genomic hybridisation; FCM, flow cytometry; FISH, fluorescence in situ hybridisation; ICM, image cytometry 
Table 1 Aneuploidy in different malignancies

\begin{tabular}{|c|c|}
\hline Malignancy & Chromosomal alteration \\
\hline Acute myeloid leukaemia ${ }^{3}$ & $\begin{array}{l}\text { Gain of chromosome } 8(13 \%) \text { and loss of } \\
\text { chromosome } 7(10 \%)\end{array}$ \\
\hline $\begin{array}{l}\text { Chronic lymphocytic } \\
\text { leukaemia }{ }^{4}\end{array}$ & Trisomy $12(30 \%)$ \\
\hline Acute lymphatic leukaemia ${ }^{5}$ & $\begin{array}{l}\text { Gain of chromosomes 4, 6, 10, 14, 17, } \\
18,20\end{array}$ \\
\hline Non-Hodgkin lymphoma ${ }^{6}$ & Trisomy 12 \\
\hline Bladder carcinoma ${ }^{6}$ & Trisomy 7, monosomy 8 \\
\hline $\begin{array}{l}\text { Malignant fibrous } \\
\text { histiocytoma }^{6}\end{array}$ & Gain of chromosome 19 \\
\hline Lung carcinoma ${ }^{7}$ & $\begin{array}{l}\text { Loss of } Y \text { chromosome and gain of } \\
\text { chromosome } 7\end{array}$ \\
\hline Breast carcinoma $^{8}$ & Trisomy $7,8,18,20$ \\
\hline Uterine carcinoma ${ }^{9}$ & $\begin{array}{l}\text { Diploid range, trisomy } 10 \text {, gain of } \\
\text { chromosomes } 2,7,12\end{array}$ \\
\hline Ovarian carcinoma ${ }^{11}$ & $\begin{array}{l}\text { Loss of chromosomes } 22,17,13,14,8 \\
\text { and gain of chromosome } 22\end{array}$ \\
\hline Prostatic carcinoma ${ }^{12} 13$ & $\begin{array}{l}\text { Normal karyotype, loss of } Y \text { chromosome } \\
\text { and gain of chromosomes } 7,14,3\end{array}$ \\
\hline Colonic carcinoma $^{14}$ & $\begin{array}{l}\text { Gain of chromosomes } 3,4,6,11,12 \text {, } \\
13,15,16 \\
\text { Loss of chromosomes } 14,15,18\end{array}$ \\
\hline
\end{tabular}

A drop of cell suspension is placed on the glass slide and stained with Giemsa, acetic orcein, or quinacrine, and studied under the microscope. ${ }^{18}$ Traditional metaphase cytogenetics are successful in the chromosomal study of haematological malignancies because the cell sample is easy to collect and to culture in aseptic condition. However, this method has many important limitations, particularly in the case of solid tumours. The specimen must be fresh, sterile, and able to be cultured in vitro, which is not always feasible in the case of solid tumours. Moreover, the dividing cell population may not be representative of the cells of interest and the chromosomes may be of poor quality. Direct touch preparations after the removal of the tumour may represent the in vivo condition, but they may contain only scant numbers of metaphase cells, which yield poor quality chromosomes for study.

\section{INTERPHASE CYTOGENETICS}

Interphase cytogenetics is remarkably free of all the shortcomings of traditional cytogenetics. FISH uses fluorescently tagged DNA probes for the visualisation of DNA sequences on metaphase spreads or interphase nuclei. ${ }^{19}$ It can be done on frozen sections or paraffin wax embedded sections of archival material. Both numerical and structural aberrations can be determined by FISH. Probes are available for the total chromosomal length, centromere, or specific loci. ${ }^{20}$ Because FISH can be performed on tissue sections, it is very useful for assessing the amount of genetic heterogeneity within tumours. The multiple samples can also be analysed rapidly by the rapid tissue array technique. ${ }^{21}$

\section{"Interphase cytogenetics is remarkably free of all the shortcomings of traditional cytogenetics"}

Multicolour FISH or spectral karyotyping are essentially similar techniques that use a combination of fluorochromes and simultaneously label all the chromosomes with different colours. ${ }^{22}{ }^{23}$ Therefore, these techniques help to visualise every chromosome in a single experiment. CGH is a relatively recent molecular cytogenetic technique, which is particularly useful in the study of solid tumours. ${ }^{24}{ }^{25} \mathrm{CGH}$ is a modified in situ hybridisation technique, in which the chromosomes are competitively hybridised with two differently labelled genomic DNA samples. One is the test (green) and other is the reference (red) DNA probe. The gain or loss of genetic material is seen as an increased or decreased ratio of green to red fluorescence, respectively, measured in a digital image morphometry machine. This method is very useful for the detection of chromosomal aberrations in solid tumours and it can be done using very small amounts of DNA.

\section{Flow cytometry}

Single cells or nuclei in fluid suspension are required for DNA FCM. The dissociated cells are stained with a DNA specific dye. These dyes bind with DNA stoichiometrically. The stained cells pass singly in front of the laser beam. The cell absorbs the light and emits fluorescence, which is measured by FCM with the help of a photomultiplier tube and digitally converted to an electronic pulse. The emitted fluorescence is proportional to the DNA content of the cells and is represented as the channel number, which is an arbitrary value depending on the machine's initial set up. The data are displayed as DNA histograms. A DNA histogram obtained in this way from peripheral blood lymphocytes should show a single peak because all the cells contain 46 chromosomes (2n). This is known as a diploid peak. Another small peak in the channel number double that of the diploid peak is also found, and this represents cells in the G2-M phase. These cells contain $2 \times 46(4 n)$ chromosomes. The cells in the process of DNA synthesis ( $S$ phase cells) will be found somewhere between the diploid and the tetraploid peaks. Any peak other than these two peaks should be considered as an aneuploid peak. ${ }^{26} 27$

\section{Image cytometry}

In ICM, the cells on the slide are stained with a DNA specific Feulgen stain, which binds with DNA stoichiometrically. The optical density of the stained nuclei is measured, and is directly proportional to the DNA content of the nuclei. ${ }^{28}$ In the case of ICM, the cells of interest can be selected under the ordinary light microscope. However, the whole process is very slow compared with FCM.

Minor chromosomal changes such as gain or loss of one or two chromosomes may not be detected by FCM and ICM.

\section{MECHANISMS OF DEVELOPMENT OF ANEUPLOIDY}

During mitosis the parent cell divides into two daughter cells with diploid numbers of chromosomes. Unequal distribution of chromosomes in the daughter cell generates aneuploidy. The various possible ways to develop aneuploidy are described below.

\section{Mitotic spindle abnormality}

Defective formation of the mitotic spindle may occur as a result of an abnormal mitotic spindle protein or supernumerary centrosomes. ${ }^{29}{ }^{30}$ Most solid cancers show centrosome amplification and aneuploidy. ${ }^{31}$ Amplification of the centrosome probably increases the frequency of abnormal mitoses and chromosome missegregation. ${ }^{32}{ }^{33}$ STK15/Aurora2 is a centrosome associated serine threonine kinase that is amplified and overexpressed in a colon cancer cell line and can induce aneuploidy. ${ }^{34}$

The product of the abnormal spindle (asp) gene is required to focus the pole of the mitotic spindle in vivo. Loss of the microtubule organisation centre is seen after the removal of Asp protein function from the cell. ${ }^{35}$

Functional defects in the mitotic spindle may also cause lagging of chromosomal movement and this may produce unequal distribution of chromosomes in the daughter cells. Unipolar movement of chromosomes in anaphase may also cause aneuploidy. 


\section{Defective attachment of chromatids}

The chromatids are attached to the mitotic spindle with the help of the kinetochore. There may not be proper separation of two sister chromatids at the kinetochore and, as a result of this non-disjunction, both the chromatids may go to one daughter cell. Merotelic attachment of chromatids at the mitotic spindle may also be a possible cause of defective segregation of chromatids at the time of anaphase. ${ }^{36}$

\section{Fusion bridging abnormality}

Rearrangement of chromosomes through breakage fusion bridge cycles may be a possible source of aneuploidy. Frequent breakage fusion bridge events were noted in human solid tumours with non-specific chromosomal aberrations. ${ }^{37}$

\section{Defective cytokinesis}

Failure of cytokinesis may produce abnormal accumulation of chromosomes in a single cell, resulting in hyperdiploid aneuploidy. ${ }^{30}$ Excessive shortening of telomeric repeats may disrupt the normal mitotic process and may be responsible for failure of cytokinesis and the development of aneuploidy.

\section{Mitotic checkpoint defects and aneuploidy}

The ultimate aim of the mitotic cell cycle is to produce two genetically identical cells from the parent one. To accomplish this, the mother cell must replicate its chromosomes exactly once before entering mitosis, and at the end of mitosis each daughter cell must receive only one copy of each chromosome. To ensure that this occurs, the cell must precisely coordinate the entire series of events. Therefore, during mitosis, cells have evolved a surveillance mechanism called the spindle assembly checkpoint, ${ }^{38-41}$ which is also known as the mitotic checkpoint. ${ }^{42}$ The spindle assembly checkpoint assesses whether the prerequisites for chromosome segregation have been met or not, and determines whether to execute or to delay chromosome segregation.

"During mitosis, cells have evolved a surveillance mechanism called the spindle assembly checkpoint, which is also known as the mitotic checkpoint"

Two major checkpoints have been noted during mitosis, one is the G2M checkpoint and the other is the metaphase checkpoint. The G2M checkpoint works as the cells enter mitosis. It closely monitors microtubule dependant events, such as the separation of duplicated centrosomes at G2, and delays the G2-M transition in the presence of microtubule poisons. The metaphase checkpoint monitors the attachment of the mitotic spindle to kinetochores. In the presence of a single unattached kinetochore, the metaphase checkpoint halts the separation of sister chromatids and thereby provides additional time for spindle attachment. Thus, the metaphase checkpoint ensures a high fidelity of chromosome separation and prevents aneuploidy during mitosis. Figure 1 explains the mechanism of the metaphase checkpoint. Six checkpoint gene products (Mad1, Mad2, Mad3, Bub1, Bub3, and Mps1) appear to operate as checkpoint sensors and signal transducers. ${ }^{43}$ It is presumed that BubRl is the functional orthologue of yeast Mad3.$^{45}$ These genes are highly conserved from yeasts to humans. In the mammalian cell, the Bubl and Mad2 proteins are present in the centromeric region during the prophase of mitosis. These proteins are detached after stable microtubule attachment during metaphase. ${ }^{42}$ The anaphase promoting complex (APC; otherwise known as cyclosome) is a 12 subunit protein complex with ubiquitin ligase activity, which causes the destruction of certain proteins by attaching them to ubiquitin. In the case of normally attached kinetochores with spindle, APC causes proteolytic destruction of securin, an anaphase inhibitor, by ligating it with ubiquitin. Separin is liberated during this interaction and this helps in sister chromatid separation and the promotion of anaphase. ${ }^{43}$ In the case of a compromised spindle, in which the kinetochore is unattached to the microtubules, an activated form of Mad2 (or other checkpoint proteins) is generated. ${ }^{45}$ This $\mathrm{Mad} 2$ molecule binds with Cdc20, an activator of anaphase promoting complex. Interaction of Mad2 with Cdc20 bound APC inhibits the ubiquitin ligase activity of APC and thus inhibits the progression of metaphase to anaphase. ${ }^{46}$

Mutations of the genes that control the human mitotic checkpoint have been noted in aneuploidy. Bubl and BubRl mutations were found in a subset of a colon cancer cell line. ${ }^{44}$ Mutations in other human mitotic checkpoint genes-Mad2 and Madl-have been noted in breast cancer and leukaemia. ${ }^{42}{ }^{47}$ These data suggest that metaphase checkpoint gene mutations significantly contribute to the development of aneuploidy.

\section{Other cell cycle checkpoints}

Inactivation of the proteins that regulate the DNA damage checkpoint and DNA replication checkpoints have been shown to cause chromosomal instability in vitro. The proteins involved in the DNA checkpoints act either as sensors of DNA damage (BRCAl, MRE1l, BLM) or as transducers (ATM, TP53, CHK2) of the DNA damage signal to the effector protein. $^{48-53}$

\section{TELOMERE DYSFUNCTION}

Pathak et al hypothesised that the genomic instability of cancer cells is caused by telomere dynamics. ${ }^{54}$ Telomeres determine the domain of the individual chromosomes within a nucleus and protect them from internal and external

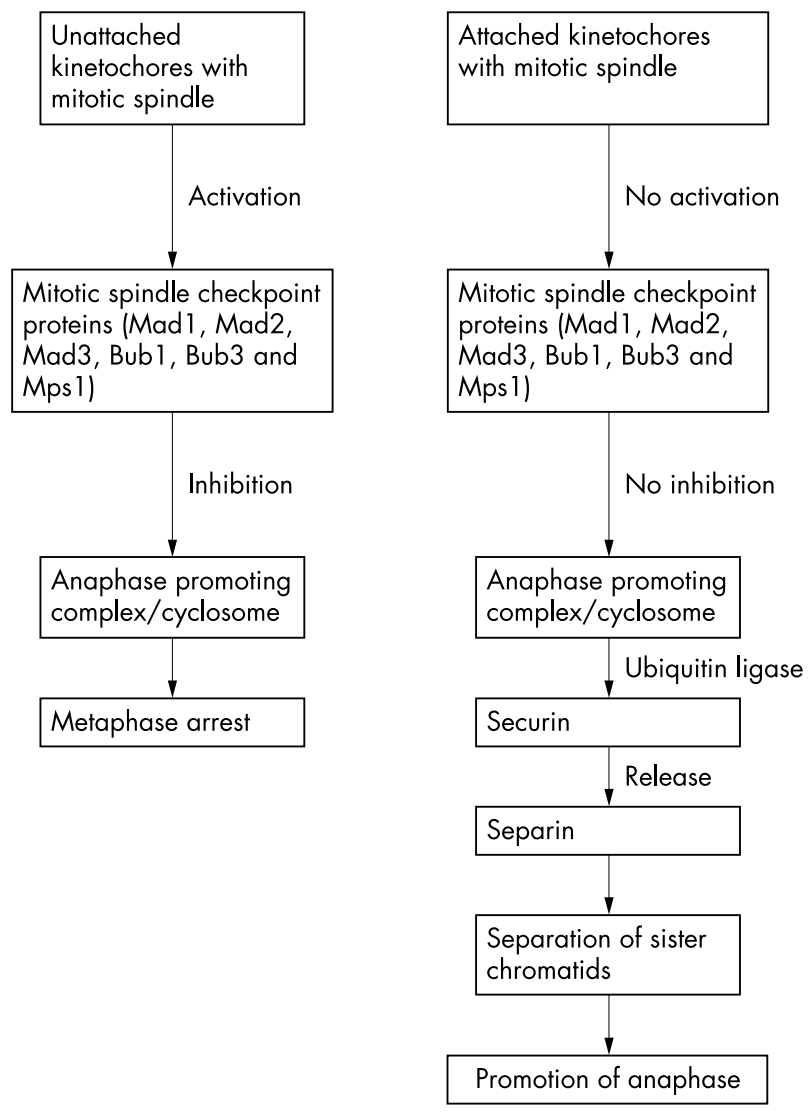

Figure 1 Schematic diagram showing the mitotic spindle checkpoint mechanism. 
challenges. Telomere erosion may destabilise the cell karyotype. In somatic cells, certain chromosomes may lose their telomeres or have a reduced amount of telomeric DNA, so that the cells may undergo translocation or other structural alterations of the DNA. Because of this loss and subsequent rearrangement of the DNA, the cells are arrested in the G2M stage of the cell cycle. The chromosomes replicate, but the cytoplasm does not, so that tetraploidy develops.

\section{ANEUPLOIDY AND CARCINOGENESIS}

It is still not clear whether aneuploidy is simply a nonspecific state that occurs spontaneously during tumour formation or whether it is involved in tumorigenesis. The following facts support an active role for aneuploidy in cancer formation. First, a specific pattern of chromosomal gain and loss occurs during the colon adenoma to carcinoma sequence. These tumour specific chromosomal abnormalities indicate that the process is not simply an epiphenomenon. ${ }^{55-58}$ Second, loss or gain of chromosomes in each generation of aneuploid cell lines is very high compared with diploid colon cancer cell lines. ${ }^{59}$ Third, mutation of mitotic checkpoint genes such as BubR1, Mad2, and Madl in colon cancer lines, breast cancer, and leukaemia, respectively, indicates the possible role of aneuploidy in carcinogenesis. ${ }^{42} 44{ }^{47}$ Fourth, there are many preneoplastic lesions that show aneuploidy $y^{60-63}$ Large cell liver dysplasia is suggested to be a preneoplastic change that progresses to hepatocellular carcinoma. Rubin et al demonstrated $100 \%$ aneuploidy in all cases of liver cell dysplasia in an image cytometric DNA ploidy study. ${ }^{60}$ Baretton et al showed frequent DNA aneuploidy in prostatic intraepithelial neoplasia with the help of a FISH technique. ${ }^{61}$ Frequent aneuploidy has also been noted in dysplastic Barrett's oesophagus, which is a preneoplastic condition. ${ }^{62}{ }^{63}$

However, there are several points against the active role of aneuploidy in cancer formation. First, aneuploidy fails to explain the slow kinetics of carcinogenesis. Second, there are a few diploid cancers and aneuploidy cannot explain the carcinogenesis in these tumours. ${ }^{1012} 13$

It is probable that aneuploidy in a cancer cell is involved in the development of a more aggressive phenotype. There are several possible ways in which aneuploidy could be involved in cancer progression, namely:

(1) Phenotypic expression of a mutated tumour suppressor gene.

(2) Polysomy of chromosomes that harbour oncogenes.

(3) Unbalanced synergistic gene.

(4) Unbalanced histones.

(5) Destabilisation of the structure of the gene by catalysing DNA break.

(6) Disturbing genes that affect the balance of the sensitive spindle apparatus.

In the aneuploid cell, there may be a loss of heterozygosity resulting in the phenotypic expression of mutated tumour suppressor genes. For example, the gain of chromosome 7 in papillary renal carcinomas reflects a duplication of the mutant MET oncogene. ${ }^{64}$ Aneuploid cells may show polysomy of chromosomes that harbour oncogenes. ${ }^{29}$ Fabarius et al suggested that aneuploidy unbalances the enzymes that synthesise and maintain DNA and nucleotide pools, and can even unbalance histones via the corresponding genes. ${ }^{65}$ DNA breaks caused by aneuploidy then initiate deletion, amplification, and intrachromosomal/interchromosomal rearrangements. Duesberg et al noted that the degree of genomic instability is proportional to the degree of aneuploidy. ${ }^{59}$ They suggested a two stage mechanism of carcinogenesis related to aneuploidy. Stage one is the generation of aneuploidy and
Take home messages

- Although aneuploidy occurs frequently in malignant tumours its role in carcinogenesis is still controversial

- Aneuploidy probably occurs as a result of failure of the spindle checkpoint or telomere dysfunction

- Several lines of evidence, such as tumour specific aneuploidy, the presence of aneuploidy in various preneoplastic conditions, the higher frequency of genetic instability in aneuploid cell lines compared with diploid cells, and mutation of mitotic checkpoint genes, suggest that aneuploidy plays an active role in carcinogenesis

- However, aneuploidy is not a prerequisite for malignancy and it is most likely that it is involved in the development of a more aggressive phenotype in the cancer cell

- The possible mechanisms involved include phenotypic expression of a mutated tumour suppressor gene, polysomy of chromosomes harbouring oncogenes, unbalanced histones, destabilisation of gene structure by catalysing DNA breaks, and disturbing genes that affect the balance of the sensitive spindle apparatus

stage two is the generation of neoplastic karyotypes by autocatalytic karyotype variation.

"The more aneuploid the karyotype, the more unstable it will be"

In stage one, both mutagenic and non-mutagenic (genotoxic and chemical) carcinogens are thought to generate aneuploidy by chemically or physically altering one or more of the chromosomes or one of the proteins of the spindle apparatus.

In stage two, aneuploidy is proposed to initiate autocatalytic karyotype variation and evolution by destabilising the karyotype. Duesberg et al suggested that aneuploidy unbalances the genes of the balance sensitive spindle apparatus, resulting in abnormal ratios of the spindle proteins, centrosomal proteins, and even abnormal numbers of chromosomes. ${ }^{59}$ Chromosome non-disjunction via an unbalanced spindle is more error prone and the degree of aneuploidy increases. The more aneuploid the karyotype, the more unstable it will be. ${ }^{59}{ }^{65}$ Over the course of time, a more lethal, aggressive, and potentially highly metastatic population develops. ${ }^{66}$ This hypothesis can explain the role of aneuploidy in most aneuploid solid malignancies; however, it fails to explain cancer progression in diploid malignancies.

\section{CONCLUSIONS}

Various factors are responsible for the development of aneuploidy. Mutation of the mitotic checkpoint genes is one of the most important factors in this respect. Telomere dysfunction is also a possible source of failure of cytokinesis, which can result in aneuploidy. Information regarding mitotic spindle checkpoint genes, the identification of aneuploidy in the colonic adenoma-carcinoma sequence, and the high degree of chromosomal instability in aneuploid cell lines have provided new insight into the role of aneuploidy in malignancy. A more complete understanding of genomic instability and aneuploidy in malignant tumours has yet to be achieved. 


\section{ACKNOWLEDGEMENTS}

Thanks to Mrs R Dey for her secretarial help.

\section{REFERENCES}

1 Nowell PC, Hungerford DA. A minute chromosome in human granulocytic leukaemia. Science 1960;132:1497.

2 Rowley JD. A new consistent chromosomal abnormality in chronic myelogenous leukemia identified by quinacrine fluorescence and Giemsa staining [letter]. Nature 1973;243:290-3.

3 Martinez-Climent JA, Lane NJ, Rubin CM. Clinical and prognostic significance of chromosomal abnormalities in childhood acute myeloid leukaemia de novo. Leukemia 1995:9:95-101.

4 Anastasi J, Le Beau MM, Vardiman JW, et al. Detection of trisomy 12 in chronic lymphocytic leukaemia by fluorescent in situ hybridisation to interphase cells, a simple and sensitive method. Blood 1992;79:1796-801.

5 Raimondi SC. Current status of cytogenetic research in childhood acute lymphoblastic leukemia. Blood 1993:81:2237-51.

6 LeBeau MM, Devita VT, Helmen S, et al, eds. Molecular biology of cancer. In Cytogenetics in cancer principles and practice of oncology, 5th ed. Philadelphia: Lippincott-Rowen, 1997:103-19.

7 Johansson $M$, Jin $Y$, Mandahl $N$, et al. Cytogenetic analysis of short term cultural squamous cell carcinoma of the lung. Cancer Genet Cytogenet 1995:81:46-55.

8 Trent J, Young J-M, Emerson J, et al. Clonal chromosomal abnormalities in human breast carcinoma II: 34 cases with metastatic disease. Genes Chromosomes Cancer 1993:7:194-203.

9 Milatvoich A, Heerema NA, Palmer CG. Cytogenetic studies of endometrial malignancies. Cancer Genet Cytogenet 1990;46:41-53.

10 Bardi G, Pandis N, Schousboe K, et al. Near-diploid karyotypes with recurrent chromosome abnormalities characterize early-stage endometrial cancer. Cancer Genet Cytogenet 1995;80:110-14.

11 Jenkins RB, Bartelt Jr D, Stalboerger $P$, et al. Cytogenetic studies of epithelial ovarian carcinoma. Cancer Genet Cytogenet 1993;71:76-86.

12 Brothman AR, Peehl DM, Patel AM, et al. Cytogenetic evaluation of 20 cultured primary prostatic tumors. Cancer Genet Cytogenet 1991;55:79-84.

13 Arps S, Rodewald A, Carl P, et al. Cytogenetic survey of 32 cancers of the prostate. Cancer Genet Cytogenet 1993;66:93-9.

14 Bardi G, Sukhikh T, Pandis N, et al. Karyotypic characterization of colorectal adenocarcinomas. Genes Chromosomes Cancer 1995; 12:97-109.

15 Johansson B, Mertens F, Mitelman F. Primary vs. secondary neoplasiaassociated chromosomal abnormalities - balanced rearrangements vs. genomic imbalances? Genes Chromosomes Cancer 1996;16:155-63.

16 Duesberg P, Stindl R, Li R, et al. Aneuploidy versus gene mutation as cause of cancer. Current Science 2001;81:490-500.

17 Sen S. Aneuploidy and cancer. Curr Opin Oncol 2000;12:82-4.

18 Vogel F, Motulsky AG. Human chromosomes. In: Human genetics, problems and approaches. Germany: Springer-Verlog Berlin Heidelberg, 1982: 18-81.

19 Wolman SR. Fluorescence in situ hybridization. A new tool for the pathologist Hum Pathol 1994;25:586-90.

20 Roylance R. Methods of molecular analysis: assessing losses and gains in tumors. Mol Pathol 2002;55:25-8.

21 Kononen J, Bubendorf L, Kallioniemi A, et al. Tissue microarrays for highthroughput molecular profiling of tumor specimens. Nat Med 1998;4:844-7

22 Schrock E, du Manoir S, Veldman T, et al. Multicolor spectral karyotyping of human chromosomes. Science 1996;273:494-7.

23 Speicher MR, Gwyn Ballard S, Ward DC. Karyotyping human chromosomes by combinatorial multi-fluor FISH. Nat Genet 1996;12:368-75.

24 Forozan F, Karhu R, Konenen J. Genome screening by comparative genomic hybridization. Trends Genet 1997;13:405-9.

25 Kallioniemi A, Kallioniemi O-P, Sudar D, et al. Comparative genomic hybridization for molecular cytogenetic analysis of solid tumours. Science 1992;258:818-21.

26 Saha I, Dey P, Vohra H, et al. Role of DNA flow cytometry and image cytometry on effusion fluid. Diagn Cytopathol 2000;22:81-5.

27 Saikia UN, Dey P, Vohra H, et al. DNA flow cytometry of non Hodgkin's lymphomas: correlation with cytologic grade and clinical relapse. Diagn Cytopathol 2000;22:152-6.

28 Dey $\mathbf{P}$, Luthra UK, Prasad A, et al. Cytological grading and DNA image cytometry of breast carcinomas on fine needle aspiration cytology smears. Anal Quant Cytol Histol 1999;21:17-20.

29 Pihan GA, Doxsey SJ. The mitotic machinery as a source of genetic instability in cancer. Semin Cancer Biol 1999;9:289-302.

30 Gisselson D. Chromosome instability in cancer: How, when, and why? Adv Cancer Res 2003;87:1-29.

31 Brinkley BR. Managing the centrosome numbers game: from chaos to stability in cancer cell division. Trends Cell Biol 2001;11:18-21.

32 Phian GA, Purohit A, Wallace J, et al. Centrosome defects can account for genetic changes that characterize prostate cancer progression. Cancer Res 2001;61:2212-19.

33 Zhou H, Kuang J, Zhong L, et al. Tumor amplified kinase STK15/BTAK induces centrosome amplification, aneuploidy and transformation. Nat Genet 1998;20:189-93.
34 Bischoff JR, Anderson L, Zhu Y, et al. A new homologue of Drosophila aurora kinase is oncogenic and amplified in human colorectal cancers. EMBO J 1998; 17:3052-65.

35 do Carmo Avides M, Glover DM. Abnormal spindle protein, Asp, and the integrity of mitotic centrosomal microtubule organizing centers. Science 1999;283:1733-5.

36 Climini D, Howell B, Maddox P, et al. Merotelic kinetochore orientation is a major mechanism of aneuploidy in mitotic mammalian tissue cells. J Cell Biol $2001 ; 153: 517-27$

37 Gisselsson D, Pettersson L, Hoglund M, et al. Chromosomal breakage-fusionbridge events cause genetic intratumor heterogeneity. Proc Natl Acad Sci U S A 2000;97:5357-62.

38 Hartwell LH, Weinert TA. Checkpoints: controls that ensure the order of cell cycle events. Science 1989;246:629-34.

39 Mclntosh JR. Mechanisms of mitosis. Trends Biochem Sci 1984;9:195-8.

40 Rudner AD, Murray AW. The spindle assembly checkpoint. Curr Opin Cell Biol 1996:8:773-80.

41 Wells WAE. The spindle-assembly checkpoint: aiming for a perfect mitosis, every time. Trends Cell Biol 1996;6:228-34.

42 Li Y, Benezra R. Identification of a human mitotic checkpoint gene: hsMAD2. Science 1996;274:246-8.

43 Hoyt MA. A new view of the spindle checkpoint. J Cell Biol 2001;154:909-12.

44 Cahil D, Lengauer C, Yu J, et al. Mutations of mitotic checkpoint genes in human cancers. Nature 1998;392:300-3.

45 Amon A. The spindle checkpoint. Curr Opin Genet Dev 1999;9:69-75.

46 Howell BJ, Hoffman DB, Fang G, et al. Visualization of Mad2 dynamics at kinetochores, along spindle fibers, and at spindle poles in living cells. J Cell Biol 2000; 150:1233-50.

47 Jin D, Spencer F, Jeang K. Human T cell leukemia virus type 1 oncoprotein Tax targets the human mitotic checkpoint protein MAD1. Cell 1998;93:81-91.

48 Bao S, Chang M-S, Auclair D, et al. RAD 17, a human homologue of the Schizosaccharomyces pombe checkpoint gene rad 17, is overexpressed in colon carcinoma. Cancer Res 1999;59:2023-8.

49 Rotman G, Shiloh Y. ATM: from gene to function. Hum Mol Genet 1998;7:1555-63.

50 Smith L. Duplication of ATR inhibits MyoD, induces aneuploidy and eliminates radiation induced G1 arrest. Nat Genet 1998;19:39-46.

51 Zhang H, Tombline G, Webeer B. BRCA1, BRCA2, and DNA damage response: collision or collusion. Cell 1998;92:433-6

52 Lane D. Awakening angels. Nature 1998:394:616-17.

53 Rouse J, Jackson SP. Interfaces between the detection, signaling and repair of DNA damage. Science 2002;297:547-51

54 Pathak S, Multani AS, Furlong Cl, et al. Telomere dynamics, aneuploidy, stem cells, and cancer [review]. Int J Oncol 2002;20:637-41.

55 Ried T, Heselmeyer-Haddad K, Blegen H, et al. Genomic changes defining the genesis, progression, and malignancy potential in solid human tumors: a phenotype/genotype correlation. Genes Chromosomes Cancer 1999;25:195-204.

56 Rooney PH, Murray GI, Stevenson DA, et al. Comparative genomic hybridization and chromosomal instability in solid tumors. Br J Cancer 1999;80:862-73

57 Hermsen M, Postma C, Baak J, et al. Colorectal adenoma to carcinoma progression follows multiple pathways of chromosomal instability. Gastroenterology 2002;123:1109-19.

58 Ried T, Knutzen R, Steinbeck R, et al. Comparative genomic hybridization reveals a specific pattern of chromosomal gains and losses during the genesis of colorectal tumors. Genes Chromosomes Cancer 1996:15:243-5.

59 Duesberg P, Rausch C, Rasnick D, et al. Genetic instability of cancer cells is proportional to their degree of aneuploidy. Proc Natl Acad Sci U S A 1998:95:13692-7.

60 Rubin EM, DeROse PB, Cohen C. Comparative image cytometric DNA ploidy of liver cell dysplasia and hepatocellular carcinoma. Mod Pathol 1994;7:677-80.

61 Baretton G, Vogt T, Valina C, et al. Prostatic cancers and potential precancerous conditions: DNA cytometric investigations and interphase cytogenetics. Verh Dtsch Ges Pathol 1993;77:86-92.

62 Persons DL, Croughan WS, Borelli KA, et al. Interphase cytogenetics of esophageal adenocarcinoma and precursor lesions. Cancer Genet Cytogenet 1998;1:11-17

63 Doak SH, Jenkins GJ, Parry EM, et al. Chromosome 4 hyperdiploidy represents an early genetic aberration in premalignant Barrett's oesophagus. Gut 2003;52:623-8.

64 Zhuang Z, Park WS, Pack S, et al. Trisomy 7-harbouring non-random duplication of mutant MRT allele in hereditary papillary renal carcinomas. Nat Genet 1998;20:66-9

65 Fabarius A, Hehlmann R, Duesberg PH. Instability of chromosome structure in cancer cells increases exponentially with degree of aneuploidy. Cancer Genet Cytogenet 2003;143:59-72.

66 Nyberg KA, Michelson RJ, Putman CW, et al. Toward maintaining the genome: DNA damage and replication checkpoints. Annu Rev Genet 2002;36:617-56. 